diagnosis, cases had at least twice as many consultations with $>1$ suggestive symptom in each 6 month period, and 20.81 times as many in the 6 months before diagnosis. Cases with combination of symptoms had an increased probability of tumor. Symptoms of brain tumor cases not observed in control children included head tilt, odd head movements, odd posture, back or neck stiffness, and unsteadiness. Recognition of unusual symptoms or specific symptom patterns and combinations is the key to identifying the child who needs prompt investigation. (Ansell P, Johnston T, Simpson J, Crouch S, Romaan E, Picton S. Brain tumor signs and symptoms: analysis of primary health care records from the UKCCS. Pediatrics Jan 2010;125(1):112-119). (Respond: Pat Ansell PhD, University of York, Dept of Health Sciences, York YO10 5DD, UK. E-mail: pat.ansell@egu.york.ac.uk).

COMMENT. Brain tumors in children are rare (2.4 per 100,000) and the average pediatrician or family practitioner will see few such cases in a lifetime of practice. With the advent of improved diagnostic methods and therapy, early diagnosis and prompt referral offer the potential for cure and a good quality of life. Knowledge of presenting symptoms of brain tumor should alert the primary care physician to suspect the diagnosis and refer the patient for neurological evaluation. (Cohen ME, Duffner PK. Brain Tumors in Children. Principles of Diagnosis and Treatment. New York; Raven Press, 1984;378). The account of the UK primary care experience emphasizes the early symptoms and signs suggestive of brain tumor and their increased prevalence and need for consultation with time. The importance of symptom combinations, unusual symptoms or specific symptom patterns is also stressed as the key to diagnosis. Early symptoms of brain tumor not addressed in the UK study include changes in affect, energy, motivation, or behavior. Indifference to playmates, listlessness, and somnolence should alert suspicion. The clinical course of a child with cerebellar medulloblsatoma, as portrayed by Cushing is worth review (Cohen and Duffner, 1984). Initial manifestations are nonlocalizing (changes in school work, vomiting, headache, enlarged head, and diplopia). These are followed by signs of a midline cerebellar syndrome, and terminally, tonsillar herniation, cerebellar fits, and decerebrate rigidity.

Seizures occurred in $50(17 \%)$ of 291 children with intracranial tumor treated at the Mayo Clinic from 1950 through 1959 (Millichap JG, Bickford RG, Miller RH, Backus RE. The EEG in children with intracranial tumors and seizures. Neurology $1962 ; 12: 329-336)$. EEG was of localizing value in $75 \%$ of supratentorial tumors and in $88 \%$ of those involving cerebral cortex. Seizures were not included in the list of symptoms observed in the UK primary care study.

\title{
SUBDURAL EFFUSION AND INFANTILE NEURONAL CEROID LIPOFUSCINOSIS
}

During the course of an ongoing NIH clinical study evaluating the benefit of cysteamine and $\mathrm{N}$-acetylcysteine in 9 patients with infantile neuronal ceroid lipofuscinosis (INCL), 4 were found to have subdural fluid collections without mass effect. Surgical drainage of fluid revealed evidence of old hemorrhage. Shearing of cortical veins as cerebral atrophy progresses is the mechanism suggested. No trauma or change in symptoms was associated with this finding. At follow-up 2.5 to 7 years later, 
the fluid collections remained stable or decreased in size. The recognition of this complication of INCL may prevent unnecessary additional investigation and intervention. (Levin SW, Baker EH, Gropman A, et al. Subdural fluid collections in patients with infantile neuronal ceroid lipofuscinosis.Arch Neurol Dec 2009;66:1567-1571). (Respond: Anil B Mukherjee MD, PhD, NIH Bldg 10, Room 9D42, 10 Center Dr, Bethesda, MD 20892. E-mail: mukherja@exchange.nih.gov).

COMMENT. INCL is a neurodegenerative disease caused by mutations in the palmitoyle-protein thioesterase-1 gene (PPT1) and resulting ceroid accumulation. Normal at birth but by 2 years the infant has complete retinal degeneration and blindness followed by seizures and psychomotor deterioration, progressing to a vegetative state by 4 years and by death. Cysteamine and $\mathrm{N}$-acetylcysteine facilitate removal of ceroids from cultured cells in patients with INCL, prompting a bench-to-bedside clinical treatment protocol.

\section{SEIZURE DISORDERS}

\section{EPILEPSY SURGERY QUALITY OF LIFE AND SEIZURE CONTROL OUTCOMES}

A consecutive, retrospective analysis of seizure control and quality of life was performed in 83 patients who underwent epilepsy surgery at Children's Hospital of Wisconsin, Milwaukee, WI. The average age at surgery was 10 years (range $<1$ year to 21 years). Surgical procedures were extratemporal focal resections (39), temporal lobectomies (19), hemispherectomies (21), and corpus callosotomies (4). Seizure outcomes were generally favorable with $68.7 \%$ class I outcome (no seizures); $12 \%$ class II (3 seizures or less per year); 19.3\% class III ( $>3$ seizures per year). Seizure freedom was highest following temporal lobectomies (84.2\%) and hemispherectomies $(76.2 \%)$. Hemispherectomy was more effective than multilobar resections. Cortical dysplasia cases did less well with a $57.5 \%$ seizure control. Infants had the lowest seizure-freedom rate at $50 \%$, attributable to frequency of multilobar resections for cortical dysplasia. Quality of life paralleled seizure outcome. Absence of defined lesion on MRI and young age should not prevent surgical evaluation of children with intractable epilepsy. (Zupanc ML, dos Santos Rubio EJ, Werner RR, et al. Epilepsy surgery outcomes: quality of life and seizure control. Pediatr Neurol Jan 2010;42:12-20). (Respond: Dr Zupanc. Department of Neurology, Children's Hospital of Wisconsin, Suite C540, 9000 W Wisconsin Ave, Milwaukee, WI 53201. E-mail: mzupanc@ mcw.edu).

COMMENT. The authors conclude that surgery for intractable epilepsy in children is superior to continued trials of antiepileptic medications. An earlier age of intervention is important since epilepsy in the developing brain may cause more permanent damage to brain circuits. Similar results were reported from the Cleveland Clinic with seizure-free outcome in $69 \%$ of adolescents, $68 \%$ children, and $60 \%$ of infants. (Wyllie E et al. Ann Neurol 1998;44(5):740-748). 